\title{
Viscosity for a Periodic Two Disk Fluid: An Existence Proof
}

\author{
Leonid A. Bunimovich ${ }^{1}$, Herbert Spohn ${ }^{2}$ \\ 1 School of Mathematics, Georgia Institute of Technology, Atlanta, Georgia 30332, USA \\ 2 Theoretische Physik, Ludwig-Maximilians-Universität, Theresienstr. 37, D-80333 München, Germany
}

Received December 10, 1994

Summary. We express the momentum current (= stress) tensor for a periodic fluid with two hard disks per unit cell in terms of a single particle billiard. We establish a central limit theorem for the time-integrated stress tensor and thereby prove the existence of a strictly positive shear and bulk viscosity.

\section{Introduction}

One of the great challenges of statistical mechanics is to prove the existence of finite (and non-zero) transport coefficients for a system of particles governed by Newton's equations of motion. For a one component fluid these transport coefficients are the shear and bulk viscosity and the thermal conductivity. There are several, presumably equivalent, ways to define them - the clearest and least ambiguous of which is through the Green-Kubo formula. Let us briefly recall the basic structure. We consider an infinitely extended, one component fluid in thermal equilibrium. The equilibrium average is denoted by $\langle\cdot\rangle$. In three dimensional physical space the fluid has five locally conserved fields: the particle density $n^{(0)}(x, t)$, the three components of the momentum density $n^{(\alpha)}(x, t), \alpha=1,2,3$, and the energy density $n^{(4)}(x, t)$, which depend on location $x \in \mathbb{R}^{3}$ and time $t \in \mathbb{R}$. [These are distributions on phase space indexed by $x, t$. Their precise form is of no importance for what follows. More details can be found in [17,21].] By the local conservation law we have, in a distributional sense,

$$
\frac{\partial}{\partial t} n^{(i)}(x, t)+\operatorname{div} j^{(\imath)}(x, t)=0
$$

$i=0, \ldots, 4$, with the local currents $j^{(\imath)}$. [Since the interaction between particles has some range, the local currents are not uniquely defined. However, the space averaged currents always are, cf. Sect. 2.] The Green-Kubo formula for the transport coefficients reads then 


$$
\Gamma_{\alpha \beta}^{(\imath \jmath)}=\frac{1}{2 k_{B} T} \int d t \int d^{3} x\left(\left\langle j_{\alpha}^{(\imath)}(x, t) j_{\beta}^{(j)}(0,0)\right\rangle-\left\langle j_{\alpha}^{(i)}(0,0)\right\rangle\left\langle j_{\beta}^{(\jmath)}(0,0)\right\rangle\right),
$$

$\alpha, \beta=1,2,3, i, j=0, \ldots, 4$, with $T$ the temperature of the fluid, where we used already the stationarity of the equilibrium measure in space-time. By rotation invariance and by time reversal symmetry in fact only three out of the $15 \times 15$ coefficients in (1.2) survive. They can be expressed by a linear combination of shear and bulk viscosity and by the thermal conductivity $[17,21]$.

Within molecular dynamics [13] it has been noted for some time that, at least in principle, transport coefficients are well defined also for systems with a finite number of particles. The main observation is that for a periodically repeated fluid the total (= space averaged) currents are meaningful and transport coefficients can still be defined via the finite volume version of (1.2). Then (1.2) equals the covariance matrix of the time-integrated total currents, normalized by $\sqrt{t}$. Thus the mathematical issue is to prove a central limit theorem for the time-integrated currents.

The simplest, yet nontrivial, case turns out to be a periodic fluid with two hard disks per unit cell. Using the well developed theory of billiards [19, 5-12] we establish a central limit theorem for the time-integrated stress (momentum current) tensor. Thereby, the first time for a mechanical system, we prove the existence of a non-zero shear and bulk viscosity. For two particles the thermal conductivity vanishes. For that case three disks per unit cell would have to be considered, which is a much tougher problem, since the boundary of the corresponding billiard has submanifolds of zero curvature.

So far, essentially the only mechanical system for which finite transport has been proved is the periodic Lorentz gas $[5-11,17]$. In this model a single point particle moves through a periodic array of convex scatterers, where it is elastically reflected. It is assumed that the time between collisions is uniformly bounded. The analogue of the total current is now merely the velocity of the particle in a unit cell with periodic boundary conditions. When integrated in time it yields the position of the particle in the periodically extended scatterer configuration. The central limit theorem for the position means that the particle diffuses in physical space. The corresponding diffusion coefficient is determined by the Green-Kubo formula, i.e., as a time integral over the velocity autocorrelation. For the viscosity we will find a similar structure with one crucial difference: the time-integrated momentum current tensor has no such direct mechanical meaning. (We should mention that Knauf [16] considers a particle in two dimensions moving in a periodic potential which has an attractive singularity. He proves the K-property for the dynamics at sufficiently high energy and a central limit theorem for the velocity.)

Our paper is organized as follows. In Sect. 2 we discuss the transport coefficients for periodic fluids in $d$ dimensions with an arbitrary number of particles per unit cell. In Sect. 3 we specialize to two hard disks. Using conservation of total momentum we arrive at a single point particle which is elastically scattered off a piecewise convex boundary. We remind the reader of some basic notions in the theory of billiards in Sect. 4 and prove the central limit theorem in Sect. 5. In an appendix by M. Fließer the viscosity is computed by molecular dynamics and compared with the one of larger size systems. 


\section{Transport Coefficients for Periodic Fluids}

We divide $\mathbb{E}^{d}$ into cells (hypercubes) of linear dimension $\ell$ and consider a fluid periodically repeated over all of $\mathbb{R}^{d}$ with $N$ particles per unit cell. They interact via a central force, $-\nabla V$. Equivalently, we consider $N$ particles, position $q_{j}$, momentum $p_{j}$, mass $m$, on the $d$-dimensional torus $\Lambda=[0, \ell]^{d}$. They interact via the pair force $F(q)=-\sum_{n \in \mathbb{Z}^{d}} \nabla V(q-n \ell)$, which is constructed as a sum over periodic images. In the short range case considered here, in fact the sum consists only of a single non-zero term.

As a distribution, the momentum density is defined by

$$
\int_{\Lambda} d^{d} x f(x) n^{(\alpha)}(x, t)=\sum_{j=1}^{N} f\left(q_{\jmath}(t)\right) p_{\jmath \alpha}(t)
$$

$\alpha=1, \ldots, d$, for all smooth test functions $f$ on $\Lambda$. We determine then the stress tensor, $\tau_{\alpha \beta}(x, t)$, through the conservation law

$$
\int_{\Lambda} d^{d} x f(x) \frac{\partial}{\partial t} n^{(\alpha)}(x, t)-\sum_{\beta=1}^{d} \int_{\Lambda} d^{d} x\left(\frac{\partial}{\partial x_{\beta}} f(x)\right) \tau_{\alpha \beta}(x, t)=0
$$

We have

$$
\begin{aligned}
\frac{d}{d t} \sum_{\jmath=1}^{N} f\left(q_{\jmath}\right) p_{\jmath}= & \sum_{\jmath=1}^{N} \frac{1}{m} p_{j} \cdot \nabla f\left(q_{j}\right) p_{j} \\
& +\frac{1}{2} \sum_{i \neq \jmath=1}^{N}\left(f\left(q_{j}\right)-f\left(q_{\imath}\right)\right) F\left(q_{\jmath}-q_{\imath}\right)
\end{aligned}
$$

where we used that $F(q)=-F(-q)$. Let us consider now one pair $i \neq j$ of particles. Then for an arbitrary smooth curve $\lambda \mapsto \gamma_{\imath \jmath}(\lambda) \in \Lambda$ with $\gamma_{\imath j}(0)=q_{\imath}, \gamma_{\imath \jmath}(1)=q_{\jmath}$ we have

$$
f\left(q_{\jmath}\right)-f\left(q_{2}\right)=\int_{0}^{1} d \lambda \dot{\gamma}_{i \jmath}(\lambda) \cdot \nabla f\left(\gamma_{\imath \jmath}(\lambda)\right) .
$$

Inserting in (2.3) and comparing with (2.2) we obtain the stress tensor

$$
\begin{aligned}
\tau_{\alpha \beta}(x, t)= & \sum_{\jmath=1}^{N} \delta\left(x-q_{j}(t)\right) \frac{1}{m} p_{\jmath \alpha}(t) p_{\jmath \beta}(t) \\
& +\frac{1}{2} \sum_{i \neq \jmath=1}^{N} \int_{0}^{1} d \lambda \dot{\gamma}_{\imath j \alpha}(\lambda) \delta\left(\gamma_{i \jmath}(\lambda)-x\right) F_{\beta}\left(q_{\jmath}(t)-q_{\imath}(t)\right) .
\end{aligned}
$$

The stress tensor depends on the choice of $\gamma_{\imath \jmath}$. This reflects that the interaction between particles is not strictly local. One conventional choice is to take the shortest straight line joining $q_{i}$ with $q_{j}$. However, for our purpose we need only the total stress tensor, which is independent of the choice for $\gamma_{\imath \jmath}$ provided $\gamma_{i j} \circ \gamma_{\jmath \imath}$ has zero winding number. We have 


$$
\begin{aligned}
\tau_{\alpha \beta}(t) & =\int_{\Lambda} d^{d} x \tau_{\alpha \beta}(x, t) \\
& =\sum_{\jmath=1}^{N} \frac{1}{m} p_{\jmath \alpha}(t) p_{j \beta}(t)+\frac{1}{2} \sum_{\imath \neq j=1}^{N}\left(q_{\jmath}(t)-q_{\imath}(t)\right)_{\alpha} F_{\beta}\left(q_{\jmath}(t)-q_{i}(t)\right) .
\end{aligned}
$$

Here $q_{j}-q_{i}$ is the shortest distance on the torus $\Lambda$.

We are now on familiar grounds. We fix the total momentum to be zero and the total energy to be $E$. Expectations with respect to the corresponding microcanonical measure are denoted by $\langle\cdot\rangle_{E, N}$. The mechanical flow on phase space is stationary under $\langle\cdot\rangle_{E, N}$. If the dynamics is ergodic and has sufficiently good mixing properties, then we expect that the time-integrated stress tensor, normalized by $\sqrt{t}$,

$$
\frac{1}{\sqrt{t}} \int_{0}^{t} d s\left(\tau_{\alpha \beta}(s)-\left\langle\tau_{\alpha \beta}(0)\right\rangle_{E, N}\right)
$$

satisfies a central limit theorem. The corresponding variance is formally given by

$$
D_{\alpha \beta, \gamma \delta}=\int d t\left(\left\langle\tau_{\alpha \beta}(t) \tau_{\gamma \delta}(0)\right\rangle_{E, N}-\left\langle\tau_{\alpha \beta}(0)\right\rangle_{E, N}\left\langle\tau_{\gamma \delta}(0)\right\rangle_{E, N}\right),
$$

$\alpha, \beta, \gamma, \delta=1, \ldots, d$. Up to conventional factors, $D$ is the viscosity tensor for the periodic fluid. For $d \geq 3$ it should be proportional to the volume $|\Lambda|=\ell^{d}$ and $D_{\alpha \beta, \gamma \delta} / 2 k_{B} T|\Lambda|$ should converge to $\Gamma_{\beta \delta}^{(\alpha \gamma)}$ of (1.2) in the infinite volume limit.

By symmetry actually most of the coefficients in (2.8) are either equal or vanish. First note that

$$
D_{\alpha \beta, \gamma \delta}=D_{\gamma \delta, \alpha \beta}=D_{\beta \alpha, \gamma \delta}=D_{\alpha \beta, \delta \gamma},
$$

since $D$ is given through a correlation and since

$$
\tau_{\alpha \beta}(t)=\tau_{\beta \alpha}(t)
$$

because $F$ is a central force. Secondly, the fluid is invariant under rotations by right angles. Let us define a linear operator, $\mathscr{Z}$, acting on the space of $d \times d$ matrices by $(\mathscr{L} A)_{\alpha \beta}=\sum_{\gamma, \delta=1}^{d} D_{\alpha \beta, \gamma \delta} A_{\gamma \delta}$. Then our discrete symmetry can be written as

$$
\mathscr{W}\left(R^{-1} A R\right)=R^{-1}(\mathscr{L} A) R
$$

for all matrices $A$ and all matrices $R$ which rotate by $90^{\circ}$. Equations (2.10) and (2.11) imply that the only nonvanishing coefficients are $D_{\alpha \beta, \alpha \beta}=D_{\beta \alpha, \alpha \beta}$ and $D_{\alpha \alpha, \beta \beta}$. Furthermore, for $\alpha \neq \beta, \gamma \neq \delta$,

$$
D_{\alpha \beta, \alpha \beta}=D_{\gamma \delta, \gamma \delta}, D_{\alpha \alpha, \alpha \alpha}=D_{\beta \beta, \beta \beta}, D_{\alpha \alpha, \beta \beta}=D_{\gamma \gamma, \delta \delta} \text {. }
$$

Thus there are in fact only three independent coefficients. Traditionally they are expressed through the shear viscosity

$$
\eta=\frac{1}{|\Lambda|} \frac{1}{2 k_{B} T} D_{12,12}
$$

and the bulk viscosity 


$$
\zeta=\frac{1}{|\Lambda|} \frac{1}{2 k_{B} T} \frac{1}{d^{2}} \sum_{\alpha, \beta=1}^{d} D_{\alpha \alpha, \beta \beta} .
$$

The third viscosity coefficient is an artifact of the periodic approximation. Under full rotation invariance (2.11) implies $2 D_{\alpha \beta, \alpha \beta}=D_{\alpha \alpha, \alpha \alpha}-D_{\alpha \alpha, \beta \beta}, \alpha \neq \beta$, in addition to (2.12). Then $\eta, \zeta$ are the only viscosity coefficients.

For the thermal conductivity we follow the same route. The total energy current turns out to be given by

$$
j_{E}(t)=\sum_{\jmath=1}^{N} \frac{1}{2 m} p_{\jmath}(t)^{2} \frac{1}{m} p_{j}(t)+\frac{1}{2} \sum_{i \neq \jmath=1}^{N}\left(q_{j}(t)-q_{i}(t)\right) \frac{1}{m} p_{\jmath}(t) \cdot F\left(q_{\jmath}(t)-q_{i}(t)\right) .
$$

Again the goal is to prove a central limit theorem for the time-integrated current,

$$
\frac{1}{\sqrt{t}} \int_{0}^{t} d s\left(j_{E}(s)-\left\langle j_{E}(0)\right\rangle_{E, N}\right)
$$

The corresponding covariance matrix is formally given by

$$
D_{\alpha \beta}^{(E)}=\int d t\left(\left\langle j_{E \alpha}(t) j_{E \beta}(0)\right\rangle_{E, N}-\left\langle j_{E \alpha}(0)\right\rangle_{E, N}\left\langle j_{E \beta}(0)\right\rangle_{E, N}\right) .
$$

Because of invariance under discrete rotations $D_{\alpha \beta}^{(E)}=\bar{\kappa} \delta_{\alpha \beta}$. Conventionally the thermal conductivity is defined by

$$
\kappa=\frac{1}{|\Lambda|} \frac{1}{2 k_{B} T^{2}} D_{11}^{(E)}
$$

$\tau_{\alpha \beta}(t)$ is even and $j_{E}(t)$ is odd under the reversal. Therefore the cross terms between (2.16) and (2.7) vanish.

\section{Viscosity for Two Hard Disks}

To prove a central limit theorem for (2.7), (2.16) is a formidable task and no general results are available. Thus we might as well try the simplest, yet nontrivial case, which clearly is $N=2$. If $p_{1}+p_{2}=0$, then

$$
m j_{E}=\frac{1}{2 m}\left(p_{1}^{2} p_{1}+p_{2}^{2} p_{2}\right)+\frac{1}{2}\left(q_{1}-q_{2}\right)\left(p_{1}+p_{2}\right) \cdot F\left(q_{1}-q_{2}\right)=0 .
$$

Thus the thermal conductivity vanishes and we are left with the viscosity only. For thermal conductivity we would have to take $N \geq 3$.

Since the total momentum is conserved, we transform canonically as

$$
\begin{aligned}
q & =q_{1}-q_{2}, & p & =p_{1}-p_{2}, \\
q_{c} & =\frac{1}{2}\left(q_{1}+q_{2}\right), & p_{c} & =\frac{1}{2}\left(p_{1}+p_{2}\right) .
\end{aligned}
$$

Then the relative motion is governed by

$$
m \frac{d}{d t} q(t)=p(t), \quad \frac{d}{d t} p(t)=2 F(q(t))
$$


where $q \in\left[-\frac{\ell}{2}, \frac{\ell}{2}\right]^{d}$ with periodic boundary conditions ( $d$-dimensional torus). Imposing the center of mass momentum $p_{c}=0$, we obtain for the stress tensor

$$
\tau_{\alpha \beta}(t)=\frac{1}{2 m} p_{\alpha}(t) p_{\beta}(t)+q_{\alpha}(t) F_{\beta}(q(t))
$$

$\alpha, \beta=1, \ldots, d$.

For the central limit theorem we certainly need the dynamics (3.3) to have good mixing properties, which we ensure by taking $V$ as a hard core potential. To simplify even further we choose $d=2$. We have then a periodically repeated fluid in the plane consisting of two hard disks of diameter $R$ per unit cell. They interact through perfectly elastic collisions. Dynamically equivalent are two disks on the two-torus. We streamline our notation. We set $p=m v$ and the mass $m=1$. To have the relative speed $|v|=1$ we set $E=\frac{1}{2 m}\left(p_{1}^{2}+p_{2}^{2}\right)=1 / 4$. The relative velocity is then specified by an angle, $\vartheta$, with $0 \leq \vartheta<2 \pi$. We take a unit two-torus, i.e., $\ell=1$. Then the only parameter left is the hard core radius $R$, which can be used to label the reduced density, i.e. density/closed packing density, according to $\rho^{*}=\sqrt{3} R^{2}$. We distinguish two dynamically very different cases, cf. Fig. 1. If $0<R<1 / 2$, then the fluid particles can easily enter the next cell and in fact may move a long distance without collision. We call this case "infinite horizon" because the corresponding one-particle billiard (3.3) has infinite horizon. If $1 / 2<R<1 / \sqrt{2}$ the disks are confined and cannot pass each other. The one-particle billiard has a "finite horizon" and its domain has a "diamond" shape. Note that because of the imposed quadratic symmetry closed packing cannot be reached. Before stating the central limit theorem, we discuss each case separately.
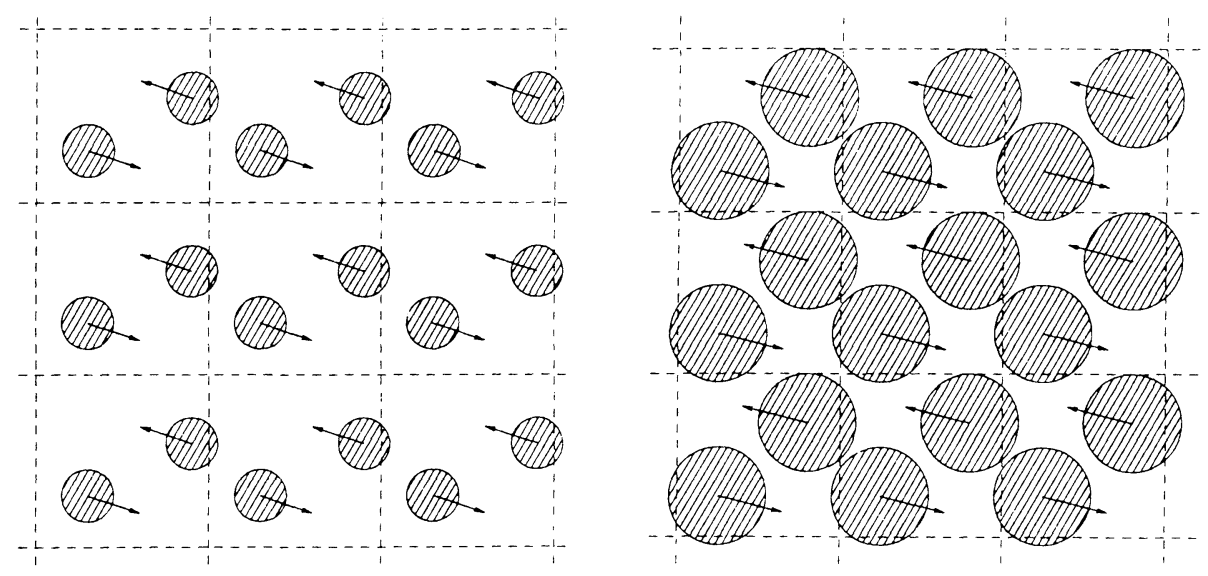

Fig. 1. Two disk fluid

3.1. Infinite horizon, $0<R<1 / 2$. The one-particle billiard is the two-torus $[-1 / 2,1 / 2]^{2}$ with a disk of radius $R$ centered at the origin, cf. Fig 2 . Let $t_{n}$ be the time of $n^{\text {th }}$ collision and $v^{\prime}\left(t_{n}\right)$ be the postcollisional, $v\left(t_{n}\right)=v^{\prime}\left(t_{n-1}\right)$ the precollisional velocity. Then the time-integrated stress tensor, $\tau_{\alpha \beta}([0, t])$, reads 

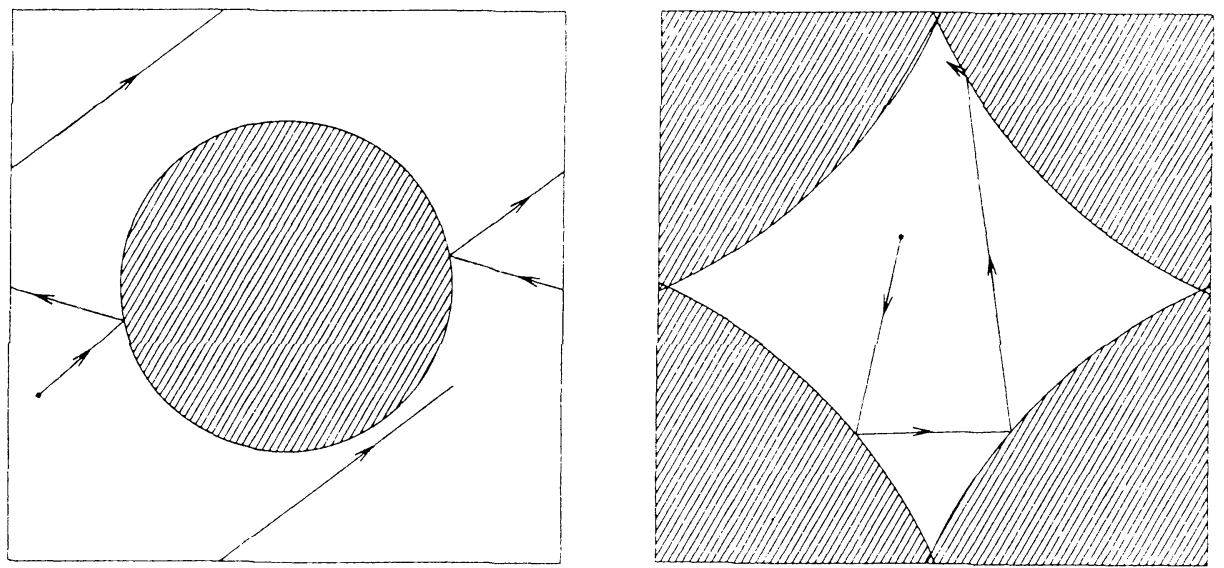

Fig. 2. Corresponding billiard problems: a) infinite horizon, b) finite horizon

$$
\begin{aligned}
\tau_{\alpha \beta}([0, t])=\frac{1}{2} & \int_{0}^{t} d s v_{\alpha}(s) v_{\beta}(s) \\
& +\frac{1}{2} \sum_{n=1}^{\infty} \chi\left(t_{n} \leq t\right) q_{\alpha}\left(t_{n}\right)\left(v^{\prime}\left(t_{n}\right)-v\left(t_{n}\right)\right)_{\beta} .
\end{aligned}
$$

The invariant measure is $Z^{-1} d q_{1} d q_{2} d \vartheta$. If we denote its average by $\langle\cdot\rangle$, then

$$
\left\langle\tau_{\alpha \beta}[0, t]\right\rangle=\delta_{\alpha \beta} t p_{D} .
$$

$p_{D}$ is sometimes called the dynamical pressure and agrees with the thermodynamic pressure in the infinite volume limit [18]. For two disks we have

$$
p_{D}=\left(4\left(1-\pi R^{2}\right)\right)^{-1} \text {. }
$$

We may simplify (3.5) by subtracting a function bounded in time. Let us again approximate the hard core by a very steep but smooth potential supported on the disk of radius $R$ and let $g(y)=y$ for $|y|<(1 / 2)-\epsilon$ and smooth on the unit circle $[-1 / 2,1 / 2]$ otherwise. Then

$$
\tau_{\alpha \beta}(t)=\frac{d}{d t}\left(1 / 2 g\left(q_{\alpha}(t)\right) v_{\beta}(t)\right)+\frac{1}{2}\left(1-g^{\prime}\left(\left(q_{\alpha}(t)\right)\right) v_{\alpha}(t) v_{\beta}(t) .\right.
$$

Let

$$
\begin{array}{r}
m_{\alpha}(n)=\operatorname{sign}\left(v_{\alpha}\left(t_{n}\right)\right) \times \text { [number of crossings of the line } q_{\alpha}=1 / 2 \\
\text { between } \left.t_{n} \text { and } t_{n+1}, t_{0}=0, t_{n+1}=t \text { if } t_{n}<t<t_{n+1}\right] .
\end{array}
$$

Then the central limit theorem has to be proved for

$$
\frac{1}{\sqrt{t}} \tilde{\tau}_{\alpha \beta}([0, t])=\frac{1}{\sqrt{t}}\left[\frac{1}{2} \sum_{n=0}^{\infty} \chi\left(t_{n} \leq t\right) m_{\alpha}(n) v_{\beta}\left(t_{n}\right)-\delta_{\alpha \beta} t p_{D}\right] .
$$

One may suspect that the central limit theorem fails for (3.9) because of the long free paths as in fact it happens for the Lorentz gas $[3,5,6,8,10,11]$. Note however that $v_{\alpha}(t) v_{\beta}(t)$ suppresses phase space volume. E.g., if in the correlation $\left\langle\tau_{12}(t) \tau_{12}(0)\right\rangle$ 
only contributions up to the first collision are taken into account, then a decay as $t^{-3}$ (integrable) results, whereas the corresponding velocity autocorrelation for the Lorentz gas decays as $t^{-1}$ (nonintegrable) only. On the other hand, for sufficiently small diameter the $45^{\circ}$ channels open up and our heuristic argument yields a $t^{-1}$ decay. We leave the central limit theorem for the infinite horizon stress tensor for future investigations.

3.2. Finite horizon, $1 / 2<R<1 / \sqrt{2}$. If we shift our coordinate system by $(1 / 2,1 / 2)$, then the one-particle billiard has diamond shape corresponding to four disks of radius $R$ centered at $( \pm 1 / 2, \pm 1 / 2)$, cf. Fig. 3. The dynamic pressure, cf. (3.6), turns out to be

$$
p_{D}=\left(1-\sqrt{4 R^{2}-1}\right)\left(4\left(1-\sqrt{4 R^{2}-1}-\pi R^{2}\left(1-\frac{4}{\pi} \arccos \frac{1}{2 R}\right)\right)\right)^{-1} .
$$

$p_{D}$ diverges at $R=1 / \sqrt{2}$ as $(4-4 \sqrt{2} R)^{-1}$ and decreases at $R=1 / 2$ as a square root. Sometimes this is considered as a precursor of the gas solid transition for the infinite hard sphere system.

As before (3.5) may be simplified by subtracting a bounded function. Since the billiard has finite horizon, $q$ is a smooth function and

$$
\tau_{\alpha \beta}(t)=\frac{d}{d t}\left(\frac{1}{2} q_{\alpha}(t) v_{\beta}(t)\right)+\left(\tilde{g}\left(q_{\alpha}(t)\right)-q_{\alpha}(t)\right) \tilde{F}_{\beta}(q(t)) .
$$

Here $\tilde{F}$ is a smoothened version of the hard core billiard force. $\tilde{g}$ appears because of the shift of the origin. We have

$$
\tilde{g}(y)-y=\left\{\begin{array}{r}
-1 / 2 \text { for } y>0 \\
1 / 2 \text { for } y<0
\end{array}\right\}=(1 / 2-\theta)(y)
$$

with $\theta$ the standard step function. The central limit theorem has to be proved for

$$
\begin{aligned}
\frac{1}{\sqrt{t}} \tilde{\tau}_{\alpha \beta}([0, t])=\frac{1}{\sqrt{t}} & {\left[\sum_{n=1}^{\infty} \chi\left(t_{n} \leq t\right)(1 / 2-\theta)\left(q_{\alpha}\left(t_{n}\right)\right)\right.} \\
& \left.\cdot \frac{1}{2}\left(v^{\prime}\left(t_{n}\right)-v\left(t_{n}\right)\right)_{\beta}-\delta_{\alpha \beta} t p_{D}\right] .
\end{aligned}
$$

3.3. Central limit theorem (finite horizon). We have $\tau_{\alpha \beta}=\tau_{\beta \alpha}$. To simplify notation we set $\boldsymbol{\tau}=\left(\tau_{1}, \tau_{2}, \tau_{3}\right)$ with

$$
\begin{aligned}
& \tau_{1}([0, t])=\frac{1}{2}\left(\tilde{\tau}_{11}([0, t])+\tilde{\tau}_{22}([0, t])\right)-p_{D} t, \\
& \tau_{2}([0, t])=\frac{1}{2}\left(\tilde{\tau}_{11}([0, t])-\tilde{\tau}_{22}([0, t])\right), \\
& \tau_{3}([0, t])=\tilde{\tau}_{12}([0, t]) .
\end{aligned}
$$

According to (2.12) the covariance matrix for $\tau$ has the form

$$
D=\left[\begin{array}{ccc}
\sigma_{1} & 0 & 0 \\
0 & \sigma_{2} & 0 \\
0 & 0 & \sigma_{3}
\end{array}\right]
$$


Then, up to dimensional factors, cf. Appendix, $\sigma_{1}=\zeta$, the bulk viscosity, $\sigma_{3}=\eta$, the shear viscosity, and $\sigma_{2}=\tilde{\eta}$ which becomes equal to $\eta$ for a rotationally invariant fluid. To establish $D>0$ as a matrix we only have to prove that for $i=1,2,3$

$$
\lim _{t \rightarrow \infty} \frac{1}{t}\left\langle\tau_{i}([0, t])^{2}\right\rangle=\sigma_{i}
$$

exists and is strictly positive, where the average is with respect to $\mu=Z^{-1} d q_{1} d q_{2} d \vartheta$.

Theorem 1. (Central limit theorem for the stress tensor). There exist a dense open subset $\mathscr{R} \subset[1 / 2,1 / \sqrt{2}]$ and variances $\left.\sigma_{\imath}: \mathscr{R} \rightarrow\right] 0, \infty[$ such that for every $R \in \mathscr{R}$ and for any real number $z$,

$$
\lim _{t \rightarrow \infty} \mu\left\{\frac{1}{\sqrt{t}} \tau_{i}([0, t])<z\right\}=\frac{1}{\sqrt{2 \pi \sigma_{i}}} \int_{-\infty}^{z} d u e^{-u^{2} / 2 \sigma_{\imath}},
$$

$i=1,2$, 3. In particular, $0<\eta, \zeta<\infty$. For every bounded open set $A \subset \mathbb{R}^{3}$ with boundary of zero Lebesgue measure we have

$$
\lim _{t \rightarrow \infty} \mu\left(\left\{\frac{1}{\sqrt{t}} \tau([0, t]) \in A\right\}\right)=\int_{A} \prod_{\imath=1}^{3} d u_{\imath} \frac{1}{\sqrt{2 \pi \sigma_{\imath}}} e^{-u_{\imath}^{2} / 2 \sigma_{\imath}}
$$

Theorem 1 establishes the existence of a strictly positive viscosity for a periodic two disk fluid. As for the Lorentz gas we also prove a bound on the stress tensor correlation function (considered only at the moments of collisions) which decays as a stretched exponential. The question whether this decay is actually exponential remains open.

\section{Theory of Billiards, Some Definitions and Results}

Let $Q$ be a bounded connected domain with a piecewise smooth (of class $C^{3}$ ) boundary $\partial Q$ belonging to the Euclidean space $\mathbb{R}^{2}$ or to a two-dimensional torus $\mathbb{T}^{2}$ with the Euclidean metric. In our case the boundary $\partial Q$ always has the specific shape of a diamond. However we will give the general definitions and results if they cannot be formulated more briefly for this special case.

Definition. A billiard in $Q$ is a dynamical system of a point particle with uniform motion inside $Q$ and with elastic collisions at the boundary $\partial Q$.

Billiards with diamond shape belong to the class of hyperbolic billiards. This means that the Lyapunov exponents are nonzero almost everywhere in phase space. Moreover diamond billiards belong to the more narrow class of hyperbolic billiards that are called dispersing (or Sinai billiards if we require also that the regular components of $\partial Q$ intersect transversally).

Regular components of the boundary $\partial Q$ are the non-self-intersecting curves $\Gamma_{i}$, $1 \leq i \leq k$, that are closed or intersect each other only at their endpoints.

We denote $\partial \tilde{Q}=\bigcup_{\imath \neq \jmath=1}^{k}\left(\Gamma_{\imath} \cap \Gamma_{\jmath}\right)$ the singular part of the boundary. In our case $\partial \tilde{Q}$ consists of four points. Correspondingly $\partial \hat{Q}=\partial Q \backslash \partial \tilde{Q}$ is the regular part of $\partial Q$.

At any regular point $q \in \partial Q$ there exists the unique internal normal vector $n(q)$. Thus the curvature $\kappa(q)$ of $\partial Q$ at such a point is uniquely defined (with respect to the 
chosen vector $n(q)$ ). In dispersing billiards the curvature $\kappa(q)$ is positive at all points of the boundary.

A billiard defines the piecewise smooth flow $\left\{S^{t}\right\}$ on its phase space $\cdot \mathbb{C}=$ $Q \times S^{1}=\{x=(q, v): q \in Q,\|v\|=1\}$. The flow $\left\{S^{t}\right\}$ preserves the Liouville measure $d \mu=c_{\mu} d^{2} q d v$, where $d^{2} q$ and $d v$ denote the Lebesgue measures on $Q$ and $S^{1}$ respectively and the constant $c_{\mu}$ is a normalization. For billiards one can naturally pass from the dynamical system with continuous time $\left\{S^{t}\right\}$ to the dynamical system with discrete time where one counts the time by the number of collisions with the boundary.

Let $M=\{x=(q, v): q \in \partial \hat{Q},(v, n(q))>0\}$, where $(\cdot, \cdot)$ is the standard inner product. Denote by $\bar{M}$ the closure of $M$ in the space $/ \mathbb{l}$. The boundary $\partial M=$ $\bar{M} \backslash M$ consists of two parts, i.e., $\partial M=S_{0} \cup V_{0}=R_{0}$, where $S_{0}=\{(q, v): q \in$ $\partial Q,(v, n(q))=0\}$, which corresponds to the "grazing" collisions with the boundary $\partial Q$, and $V_{0}=\{(q, v): q \in \partial \tilde{Q}\}$, which corresponds to the singular points ("corners") of $\partial Q$. We denote by $T$ the map on $\bar{M}$ induced by the flow $\left\{S^{t}\right\}$.

We introduce in $\bar{M}$ the coordinates $(r, \varphi)$, where $r$ is the parameter of the length of an arc on the curve $\partial Q$ and $\varphi$ is the angle between the vectors $v$ and $n(q)(-\pi / 2 \leq$ $\varphi \leq \pi / 2)$. In our case in these coordinates $\bar{M}$ is the union of four rectangles. The map $T$ preserves the measure $d \nu=c_{\nu} \cos \varphi d r d \varphi$, where $c_{\nu}$ is the normalization. Let us denote by $\tau_{+}(x)=\tau(x)$ and $\tau_{-}(x)$ the first positive and the first negative moment of collision of the trajectory of $x \in M$ with the boundary. Thus $T^{ \pm 1} x=S^{\tau_{ \pm}(x)+0} x$.

The mappings $T$ and $T^{-1}$ are piecewise smooth. The map $T\left(T^{-1}\right)$ has singularities on the set $T^{-1} R_{0}\left(T R_{0}\right)$. We denote $R_{i}=T^{i} R_{0}$ and $R_{m, n}=\bigcup_{\imath=m}^{n} R_{\imath}$, $-\infty \leq m \leq n \leq \infty$. It is easy to see that the set of singularities for $T^{n}\left(T^{-n}\right)$, $n \geq 1$, is $R_{-n, 0}\left(R_{0, n}\right)$. The set $R_{-\infty, \infty}$ consists of a countable number of curves that we call discontinuity curves.

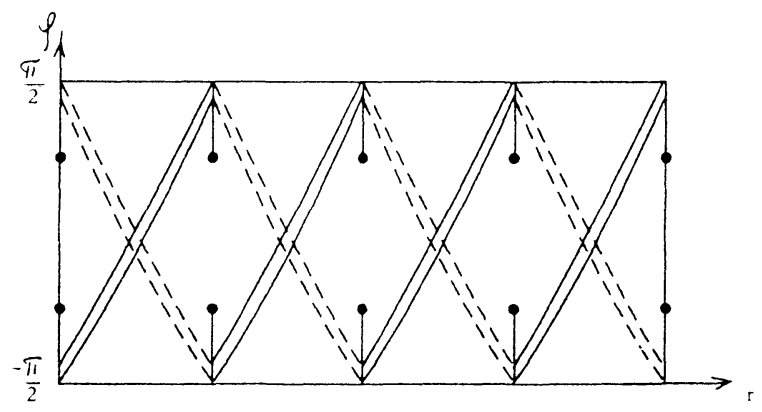

Fig. 3. Structure of discontinuity and of singularity curves for a diamond

The following lemma (see $[8,10]$ ) is simple but very important for the understanding of the structure of the set of discontinuity curves.

Lemma 4.1. Let $\Gamma \subset R_{m}\left(R_{-m}\right), m \geq 1$. Then its endpoints $\partial \Gamma$ belong to the set $R_{0, m}\left(R_{-m, 0}\right)$.

A point $x$ will be called a multiple point if it belongs to more than one discontinuity curve. A rank of a multiple point $x$ is $\min \left\{n: x \in R_{-n, n}\right\}$. Consider a curve $\gamma \subset \bar{M}$ of class $C^{1}$. A curve $\gamma$ is called increasing (decreasing) if it can be defined by an equation $\varphi=\varphi(r)$ such that $d \varphi / d r>0(d \varphi / d r<0)$. The property to be an 
increasing (decreasing) curve is preserved under the action of $T\left(T^{-1}\right)$. We call a curve $\gamma m$-increasing one ( $m$-decreasing), $m \geq 1$, if $T^{-m} \gamma\left(T^{m} \gamma\right)$ is an increasing (decreasing) smooth curve. All discontinuity curves from the set $R_{1, \infty}\left(R_{-\infty,-1}\right)$ are the increasing (decreasing) ones. Let $\gamma$ be an increasing (decreasing) curve with the equation $\varphi=\varphi(r)$. We introduce the $p$-length of $\gamma$ via the formula $p(\gamma)=\int_{\gamma} \cos \varphi d r$.

All increasing (decreasing) curves are stretched (in the sense of $p$-length) under the action of the map $T\left(T^{-1}\right)$. This implies the hyperbolicity of the map $T$.

Hyperbolicity means that for almost every point $x \in M$ there exist two $C^{1}$-curves $\gamma^{(u)}(x)$ and $\gamma^{(s)}(x)$ such that $\left.T^{n}\right|_{\gamma^{(s)}(x)}\left(\left.T^{-n}\right|_{\gamma^{(u)}(x)}\right)$ is a smooth map for any $n \geq 1$ and $\lim _{n \rightarrow \infty} p\left(T^{n} \gamma^{(s)}(x)\right)=0, \lim _{n \rightarrow \infty} p\left(T^{-n} \gamma^{(u)}(x)\right)=0$. Such curves $\gamma^{(u)}(x), \gamma^{(s)}(x)$ are called respectively, a local unstable manifold and a local stable manifold of the point $x$ and are denoted LUM and LSM of $x$. At some point of Sect. 5 we shall use the notion of homogeneous local manifolds (HLSM and HLUM). These are segments of LSM and LUM which are partitioned into not "too many" pieces by discontinuity curves originating from the dynamics (see Definition 3.3 in [8]).

In [7, 9-12] some special Markov approximations of the billiard dynamics were constructed. These approximations allow one to study the statistical properties of the corresponding billiards, e.g., to estimate the rate of the decay of correlations, to prove the central limit theorem, etc.

To get such approximations one needs to construct the special partitions of the phase space $M$ (Markov partitions [7,9]) or of some subset of the measure $1-\alpha(n)$, where $\lim _{n \rightarrow \infty} \alpha(n)=0$ and $n$ is a number of collisions (Markov sieves [8-10]).

In this paper we do not need to give the exact (and long) definitions of a Markov partition or of a Markov sieve. We will formulate here only the conditions that are needed for their construction and describe how the elements of this partition look in our case.

Condition A. All regular components of the boundary $\partial Q$ intersect transversally. This condition is ensured by choosing $R>1 / 2$ in the two disk fluid.

Condition B. For any $m \geq 1$ a number of discontinuity curves of the set $R_{-m, m}$ which contain any point $x \in \bar{M}$ is bounded from above by $K_{0} m$, where $K_{0}=K_{0}(Q)$ is some constant. This condition will be proven in Sect. 5 for generic domains of diamond shape.

A parallelogram is a subset $U \subset \bar{M}$ such that for any two points $x, y \in U$ the intersections $\gamma^{(s)}(x) \cap \gamma^{(u)}(y)$ and $\gamma^{(u)}(x) \cap \gamma^{(s)}(y)$ each contain only one point and these two points belong to $U$ too. Thus a parallelogram is a set with a structure of a direct product. Indeed to obtain a parallelogram $U \ni x$ one should take $\gamma^{(s)}(x)$ and $\gamma^{(u)}(x)$ and then take all intersections of sufficiently long LUMs and LSMs that intersect $\gamma^{(s)}(x)$ and $\gamma^{(u)}(x)$ respectively.

Let $A$ be any measurable subset of $\bar{M}$. Denote $\gamma_{A}^{(s, u)}(x)=\gamma^{(s, u)}(x) \cap A$. We shall call a parallelogram $U O$-homogeneous if for any point $x$ of it the set $\gamma_{U}^{(u)}(x)$ $\left(\gamma_{U}^{(s)}(x)\right)$ is contained in HLUM (HLSM). Essentially it means that the coefficients of expansion (contraction) at different points of such parallelogram do not differ too strongly from each other.

We will call a rectangle a domain $K \subset M$, which is bounded by two LUM and by two LSM such that the ends of each LUM belong to LSMs and vice versa. The 
corresponding LUMs (LSMs) are called $u$-boundaries ( $s$-boundaries) of $K$. For any parallelogram $U \subset \bar{M}$ one can find the minimal rectangular $K(U) \supset U$. We will call $K(U)$ the support of a parallelogram $U$. By the $u$-boundary ( $s$-boundary) of a parallelogram $U$ we mean the $u$-boundary ( $s$-boundary) of $K(U)$.

\section{Proof of Theorem 1}

In this section we verify that the shape of our billiard is generic, that the components of the stress tensor are the generic functions and that moreover the full stress tensor is the generic too. All these notions of genericity will be defined exactly below. Actually they mean that our generic domain and the functions that we are interested in satisfy to the conditions under which the central limit theorem was proven in [8].

Lemma 5.1. A generic domain with diamond shape satisfies Condition B.

Proof. As has been explained in Sect. 4 the set $R_{-m, m}$ is formed by the images (under $T^{k},-m \leq k \leq m$ ) of the singularities of the boundary $\partial Q$ and of the trajectories tangent to $\partial Q$.

The set $R_{-1,1}$ consists of a finite number of curves. These curves are represented in Fig. 3.

Thus Lemma 8.6 of [8] holds for the discontinuity curves of our billiard. To make the exposition self-contained we repeat here its statement.

Lemma 5.2. For any multiple point $x \in M \backslash S_{0}$ and for any integer $m>0$ there exists a neighborhood $U(x)$ such that

(i) The closure $\overline{U(x)}$ does not contain multiple points, besides $x$ itself, with ranks that does not exceed $m$.

(ii) There exists the single curve $\Gamma_{+}(x) \in R_{n}(x)\left(I_{-}^{\prime}(x) \in R_{n}(x)\right)$, among all discontinuity curves which pass through $x$, such that $\Gamma_{+}(x)\left(\Gamma_{-}(x)\right)$ divides $U(x)$ into two semi-neighborhoods $U_{1}^{+}(x)$ and $U_{2}^{+}(x)\left(U_{1}^{-}(x)\right.$ and $\left.U_{2}^{-}(x)\right)$.

(iii) All decreasing (increasing) discontinuity curves, besides $\Gamma_{-}(x)\left(\Gamma_{+}(x)\right)$, containing $x$, intersect only one of the semi-neighborhoods $U_{1}^{+}(x)$ or $U_{2}^{+}(x)\left(U_{1}^{-}(x)\right.$ or $\left.U_{2}^{-}(x)\right)$ (see Fig. 4).

The new feature of diamonds in comparison with the domains studied in [8] is the presence of the singular part $\partial \tilde{Q}$ of the boundary. Thus we should take care on trajectories that intersect $\partial \tilde{Q}$. Let $\pi(T x) \subset \partial \tilde{Q}$. Consider a neighborhood $U(x) \ni x$. Its image $T U(x)$ becomes "broken" into two semi-neighborhoods, each of which has the boundary that belongs to $\pi^{-1}(\partial \tilde{Q})$.

These semi-neighborhoods then are "glueing" into the "new" neighborhoods. Thus, in a "bad" case two semi-neighborhoods with many singularity curves can be glued together. Therefore, in this case a number of singularity curves passing through a point, in principle, can grow exponentially with a number of iterations.

In a generic case, however, not more than two singularity curves can intersect at one point. An union of the angles of a diamond, when it is true, form an open and everywhere dense subset in the interval $\left(0,180^{\circ}\right)$. In fact, any triple intersection of singularity curves can be destroyed by a small perturbation of the angle of a diamond.

This implies Lemma 5.1. 


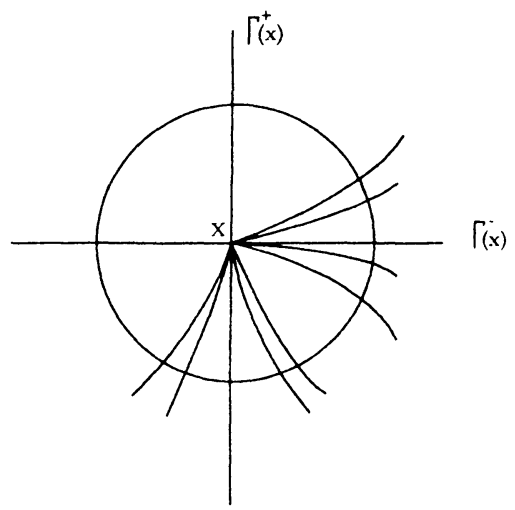

Fig. 4. Local structure of discontinuity curves

Remark 5.1. Lemma 5.1 does not provide any concrete examples of diamonds that satisfy Condition B. It states, however, that it is fulfilled for "almost all" diamonds. It is easy to check, however, that diamonds with the angles of the form $180^{\circ} / n$ or $180^{\circ} /\left(n+\frac{1}{2}\right)$, where $n>0$ is an integer satisfy Condition B.

The proof of Theorem 1 follows the same line of reasoning as given in $[8,10]$, which is not repeated here except for one assertion where details were not supplied in [8]. However to make our paper reasonably self-contained we state the main propositions providing outlines if necessary.

As standard the first step is the reduction of the dynamical system with continuous time, $t$, to discrete time counted in number of collisions, $n$. To make such reduction one needs to prove the central limit theorem for the function $\tau(x)-\langle\tau\rangle$, i.e., for the fluctuations of the time between collisions. Since our class of billiards belong to dispersing billiards with finite horizon the following statement holds (see e.g., [8]).

\section{Lemma 5.3.}

$$
\lim _{n \rightarrow \infty} \nu\left\{\frac{\tau(x)+\cdots+\tau\left(T^{n-1} x\right)-n\langle\tau\rangle}{\sigma_{\tau} \sqrt{n}}\right\}=\frac{1}{\sqrt{2 \pi}} \int_{-\infty}^{x} d u e^{-u^{2} / 2},
$$

where $\sigma_{\tau}^{2}=\sum_{m=-\infty}^{\infty}\left\langle(\tau(x)-\langle\tau\rangle)\left(\tau\left(T^{m} x\right)-\langle\tau\rangle\right)\right\rangle>0$.

Therefore in Theorem 1 we may replace $t$ by $\left\langle\tau>n\right.$. Let $\tau_{i}(n, x), i=1,2,3$, be the value of $\tau_{i}([0, t])$ immediately after the $n^{\text {th }}$ collision. Using Lemma 5.3 it suffices then to show that

$$
\begin{aligned}
\lim _{n \rightarrow \infty} \nu\left(\left\{(<\tau>n)^{-1 / 2}\left(\tau_{1}(n, x), \tau_{2}(n, x), \tau_{3}(n, x)\right) \in A\right\}\right) & \\
& =\int_{A} d q_{1} d q_{2} d q_{3} f_{1}\left(q_{1}, q_{2}, q_{3}\right),
\end{aligned}
$$

where $f_{1}\left(q_{1}, q_{2}, q_{3}\right)$ is the Gaussian distribution in Theorem 1.

According to (3.13), (3.14) we have

$$
\tau_{\imath}(n, x)=\Delta_{i}(x)+\Delta_{\imath}(T x)+\cdots+\Delta_{i}\left(T^{n-1} x\right) .
$$

It is easy to see that $\Delta_{\imath}(x), i=1,2,3$, is a piecewise Hölder continuous function. Furthermore by construction $\left\langle\Delta_{\imath}(x)\right\rangle=0$. Therefore to prove Theorem 1 one needs to show that 


$$
\sigma_{i}=\sum_{n=-\infty}^{\infty}\left\langle\Delta_{i}(x) \Delta_{\imath}\left(T^{n} x\right)\right\rangle>0 .
$$

It is well known (see e.g., [8]) that a degeneracy of the limiting distribution for $\Delta_{i}, i=1,2,3$ is equivalent to the existence of a function $G_{\imath}(x) \in L^{2}(M, \nu), i=1,2,3$ such that

$$
\Delta_{\imath}(x)=G_{\imath}(T x)-G_{\imath}(x) .
$$

The following statement is crucial for the proof of Theorem 1:

Lemma 5.4. For any function $\Delta_{i}(x), i=1,2,3$, there exist periodic point $y_{i} \in M$, $i=1,2,3$ such that

$$
S_{i}\left(y_{i}\right)=\Delta_{i}\left(y_{\imath}\right)+\Delta_{\imath}\left(T y_{i}\right)+\cdots+\Delta_{\imath}\left(T^{k_{i}-1}\right) \neq 0,
$$

where $k_{\imath}, i=1,2,3$ are the periods of the points $y_{\imath}, i=1,2,3$ respectively.

Proof. We only use the two periodic trajectories represented in Fig. 5a),b).

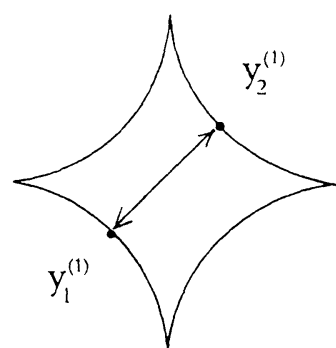

a)

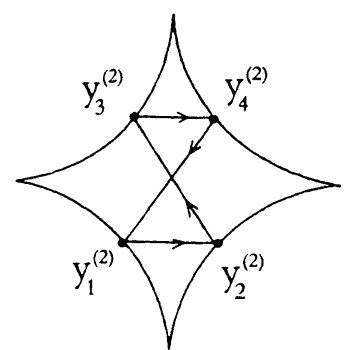

b)

Fig. 5. Periodic trajectories of a diamond billiard

The existence of the period 2 trajectory (Fig. 5a)) is obvious. One checks that $\tau_{1}$ and $\tau_{3}$ integrated over this orbit is strictly positive for all $1 / 2<R<1 / \sqrt{2}$. The existence of the period 4 orbit (Fig. $5 b$ )) follows from continuity: A trajectory parallel to the 1 -axis will cut the 1 -axis to the right of 0 after a collision at $45^{\circ}$, whereas it will cut the 1-axis to the left of 0 after a collision at the cusp. A somewhat more tedious calculation shows that $\tau_{2}$ integrated over this period 4 orbit is strictly positive for $1 / 2<R<1 / \sqrt{2}$.

The proof of the following statement (for the general dispersing billiards) is contained in [8].

Lemma 5.5. Let $y_{0}$ be a periodic point of a discrete dynamical system generated by a dispersing billiard. For any $\epsilon_{0}>0$ there exists a O-homogeneous parallelogram $U_{0} \ni y_{0}$ such that $y_{0} \notin \partial K\left(U_{0}\right)$ and $\nu\left(U_{0}\right) / \nu\left(K\left(U_{0}\right)\right)>1-\epsilon_{0}$.

We fix now the periodic point $y_{0}$ considered in Lemma 5.4 and the parallelogram $U_{0}$ from Lemma 5.5 for some small $\epsilon_{0}$. If $\epsilon_{0}$ is sufficiently small then $T^{k} U_{0}$ is also the $O$-homogeneous parallelogram and $T^{k} U_{0} \cap U_{0}$ is the correct intersection, i.e., it is bounded by the stable boundary of $K\left(U_{0}\right)$ and by the unstable boundary of $K\left(T^{k} U_{0}\right)$. 
Let $G_{\imath}(x) \in L_{2}(M, \nu)$ be the homology of a function $\tau_{i}(x), i=1,2,3$. That means $\tau_{\imath}(x) \equiv G_{\imath}(T x)-G_{i}(x)$ almost everywhere in $M$. The functions $G_{\imath}(x), i=1,2,3$ are measurable and integrable ones. Thus the following statement holds (see [8]).

Lemma 5.6. For any $\epsilon_{1}>0$ one can find a O-homogeneous parallelogram $U_{1}$ such that $\nu\left(U_{1}\right) / \nu\left(K\left(U_{1}\right)\right)>1-\epsilon_{1}$ and some real number $g$ such that

$$
\frac{\nu\left\{x \in U_{1}:|G(x)-g|>\epsilon_{1}\right\}}{\nu\left(U_{1}\right)}<\epsilon_{1},
$$

where instead of $G(x), g$ one should substitute $G_{i}(x), g_{i}, i=1,2,3$ respectively.

The first condition of Lemma 5.6 means that the parallelogram $U_{1}$ is "sufficiently" dense and the second one shows that the function $G(x)$ in $U_{1}$ can be approximated by a constant.

The following assertion was not proven (and not formulated explicitly in [10]). Let us define the "almost" first return mapping $T_{1}$ on $U_{1}$ via the following procedure.

For a point $x \in U_{1}$ we take on its positive semi-trajectory the first point $T^{n} x \in U_{0}$. Now we take the first point $T^{n+k} x$ on the positive semi-trajectory of the point $T^{n} x$ such that $T^{n+k} \in U_{1}$. Moreover we demand that $T^{\ell} x \notin U_{1}$ for $\ell=1,2, \ldots, n-1$.

We define now $T_{1} x=T^{n+k} x$. Thus the map $T_{1}: U_{1} \rightarrow U_{1}$ is defined on the subset of all such points of $U_{1}$ which return to $U_{1}$ and besides do visit the neighborhood $U_{0}$ before that.

Lemma 5.7. One can choose $U_{1}$ so small that the map $T_{1}: U_{1} \rightarrow U_{1}$ would be invertible and preserves the measure $\nu$.

Proof. Strictly speaking a map $T_{1}$ (and $U_{1}$ ) depends on the function $\left(G_{\imath}(x), i=\right.$ 1,2,3. We'll give the proof for an abstract function $G(x) \in L^{2}(M, \nu)$. We take a parallelogram $U_{1}$ that satisfies the conditions of Lemma 5.6. Let $n_{1}(x) \geq 0, x \in U_{1}$, be a minimal number such that $T^{n_{1}(x)} \in U_{1}$. Define

$$
U_{1}^{\prime}=\left\{x: x \in U_{1}, n_{1}(x)>0, \exists n_{0}(x)<n_{1}(x) \text { such that } T^{n_{0}(x)} x \in U_{0}\right\} .
$$

From the ergodicity of $T$ it follows that $\mu\left(U_{1}^{\prime}\right)>0$ if $U_{1}$ has been chosen sufficiently small.

We choose now a density point $z \in U_{1}^{\prime}$ such that $z$ is nonperiodic and moreover its trajectory $\left\{T^{m} z\right\},-\infty<m<\infty$, never hits the singularity set of a dynamical system under consideration.

Let $n>0$ be the minimal integer such that $T^{n} z \in U_{00}$, where $U_{00} \subset U_{0}$ is the subset that contains all such points $x \in U_{0}$ that $U_{0} \cap \gamma^{(u)}(x)\left(U_{0} \cap \gamma^{(s)}(x)\right)$ is the dense subset of $\gamma^{(u)}(x)\left(\gamma^{(s)}(x)\right)$. (More precisely a point $y \in U_{0}$ belongs to $U_{00}$ iff for the unstable fiber $\gamma^{(u)}(y)$ through $y$ the intersection $U_{0} \cap \gamma^{(u)}(y)$ has a density point at $y$ and the same is true for the stable fiber through $y$.)

Take now a very small parallelogram $U$ centered at the point $z$ such that $\left.T^{n}\right|_{U}$ is continuous. Besides since $z$ is aperiodic, the first $n$ iterates of $U$ do not send $U$ back to $U$ because $U$ was chosen to be small enough.

Consider now $T^{n} U$. It is a parallelogram and its center $T^{n} z \subset U_{0}$. Moreover $U_{0}$ is very dense around that point $T^{n} z$.

We take the intersection $T^{n} U \cap U_{0}$. It's also a parallelogram. If $U$ is small enough, then $T^{n} U$ is also small so that the intersection is a very dense parallelogram at $T^{n} z$. 
By taking it $k$ iterations backward in time, we obtain the parallelogram $U$ which we need. Thus Lemma 5.7 is proven.

The end of the proof of Theorem 1 proceeds in completely analogy to [10].

Let us define now two maps, $\phi$ and $\phi_{1}$ on $U_{1}$. Take $x \in U_{1}$ and let $T^{n_{0}} x \in U_{0}$ be the first point of its trajectory in $U_{0}$, and let $T^{n_{1}} x=T_{1} x$, where $n_{1}>n_{0}$. Denote $\tilde{x}=\gamma^{(u)}\left(T^{n_{0}+k} x\right) \cap \gamma^{(s)}\left(T^{n_{0}} x\right)$ and define $\phi x=T^{-n_{0}-k} \tilde{x}$ and $\phi_{1} x=T^{n_{1}-n_{0}} \tilde{x}$.

It is easy to see that $\phi x$ is a point which belongs to the same HLUM as $x$ and $\phi_{1} x$ belongs to the same HLSM as $T_{1} x$. Moreover, $T^{n_{1}+k}(\phi x)=\phi_{1} x$.

We put

$$
S_{\imath}(x)=\Delta_{i}(x)+\cdots+\Delta_{i}\left(T^{n_{1}-1} x\right)
$$

and

$$
S_{i}^{\prime}(\Phi x)=\Delta_{i}(\phi x)+\Delta_{\imath}(T(\phi x))+\cdots+\Delta_{\imath}\left(T^{n_{1}+k-1}(\phi x)\right), \quad i=1,2,3 .
$$

Lemma 5.8. $\left|S^{\prime}(\phi x)-S(x)\right| \geq S_{0}-\epsilon_{2}$, where $\epsilon_{2} \rightarrow 0$ as diam $U_{0} \rightarrow 0$.

In fact the trajectories of $x$ and $\phi x$ are close during the first $n_{0}$ iterations. Further, the trajectories of $T^{n_{0}} x$ and $T^{n_{0}+k}(\phi x)=\tilde{x}$ are close during $n_{1}-n$ iterations. The piecewise Hölder continuity of $\Delta_{\imath}(x)$ on the continuity set of $T$ implies that the corresponding sums, occurring in the sums $S_{\imath}(x)$ and $S_{\imath}^{\prime}(\phi x)$ are close to each other.

There remain exactly $k$ terms in the sum $S_{i}^{\prime}(\phi x)$ not accounted for, coming from the images of $T^{n_{0}}(\phi x)=T^{-k} \tilde{x}$. But these images approximate the periodic orbit of $y_{0}$. Hence the corresponding sum of values of $\Delta_{i}$ is close to $S_{0}$ and the lemma has been proven for any $i=1,2,3$. Lemma 5.8 implies the inequality

$$
\left|G\left(T_{1} x\right)-G(x)\right|+\left|G\left(\phi_{1} x\right)-G(\phi x)\right| \geq S_{0}-\epsilon_{2} .
$$

It remains to prove that for the majority (with respect to the measure $\nu$ ) of points $x \in U_{1}$ their images $\phi x$ and $\phi_{1} x$ coincide in $U_{1}$, and that the sets of these images $\{\phi x\}$ and $\left\{\phi_{1} x\right\}$, have relatively large measure (>const $\left.V\left(U_{1}\right)\right)$. Then for such points $\phi_{1} x=T_{1}(\phi x)$ and together with (5.2) this leads to a contradiction with the second estimate in Lemma 5.6.

Consider now the sets $\phi U_{1}$ and $\phi_{1} U_{1}$. Since $T^{n_{0}} x \in U_{0}$ the set $\gamma_{0}=\gamma_{K\left(U_{0}\right)}^{(u)}\left(T^{n_{0}} x\right)$ is an HLUM stretched on $K\left(U_{0}\right)$. Its inverse image $T^{-n_{0}} \gamma_{0}$ is a subsegment of the HLUM $\gamma_{K\left(U_{1}\right)}^{(u)}(x)$. Since for typical $x$ the parallelogram $U_{1}$ is dense, the set $T^{n_{0}} U_{1}$ has a high density on $\gamma_{0}$. The map transforming $T^{n_{0}} x$ to $T^{k} \tilde{x}$ is a contraction of the HLUM $\gamma_{0}$ with a coefficient close to $\Lambda_{1}=\Lambda_{k}^{u}\left(y_{0}\right)$, where $\Lambda_{k}^{u}\left(y_{0}\right)$ is the coefficient of expansion of the LUM $\gamma^{u}\left(y_{0}\right)$ at $y_{0}$ under $T^{k}$. Indeed $\Lambda_{1}$ is the coefficient of contraction of the LUM $\gamma^{(u)}\left(y_{0}\right)$ at the point $y_{0}$ under $T^{-k}$. Since $U_{0}$ and $U_{1}$ are the $O$-homogeneous parallelograms, the conditional measures of $U_{1}$ and $\phi U_{1}$ on $T^{-n_{0}} \gamma_{0}$ are in the ratio 1 : (const $\left.\Lambda_{1}^{-1}\right)$. This gives the required estimate $\nu\left(\phi U_{1}\right) \geq$ const $\Lambda_{1}^{-1} \nu\left(U_{1}\right)$. Similarly, $\nu\left(\phi_{1} U_{1}\right) \geq$ const $\Lambda_{2}^{-1} \nu\left(U_{1}\right)$, where $\Lambda_{2}=\Lambda_{k}^{s}\left(y_{0}\right)$ is the coefficient of contraction of the $\operatorname{LSM} \gamma^{s}\left(y_{0}\right)$ at $y_{0}$ under $T^{k}$.

This completes the proof of Theorem 1. 


\section{Conclusions}

We have proven that for a periodic two disk fluid in two dimensions the transport coefficients of shear and bulk viscosity exist provided the disk diameter is sufficiently large. This geometrical condition allows us to reduce the problem to the study of a two-dimensional billiard with finite horizon. In the case of an infinite horizon one would have to estimate the decay of correlations for the billiard flow rather than for the corresponding dynamical system with discrete time.

Amongst the possible generalizations the most immediate one is to prove the same result for a $d$-dimensional $(d>2)$ periodic two particle fluid with finite horizon. Some techniques to deal with such a problem are provided in [11], which however covers smooth boundaries only. A further generalization of interest refers to the heat conductivity. Here at least three particles per unit cell have to be considered. Thus first a Markov sieve (or a Markov partition) has to be constructed for a system of three disks on a torus. At the moment such a construction is not available, but the problem is under investigation.

As to be explained in the Appendix below, the shear viscosity of the $N=2$ periodic hard disk fluid compares surprisingly well with the Enskog theory and with numerical results for the $N=108,500,4000$ periodic hard sphere fluids. Such little $N$ dependence must mean that to a good approximation small subsystems are statistically independent. The stress-stress correlation of a large system decays on the same time scale as the one of the $N=2$ system. Dissipation is essentially local.

\section{Appendix: Viscosity from Molecular Dynamics}

\section{by Martin Fließer}

Theoretische Physik, Ludwig-Maximilians-Universität München, München, Germany

The proof of Theorem 1 gives, if at all, very little information on the actual value of the viscosity. For this one has to determine numerically the averages $\left\langle\tau_{j}\left([0, t]^{2}\right\rangle\right.$, $j=1,2,3$. By Monte-Carlo we generate $10^{5}$ initial phase points distributed according to $\nu$, i.e. uniformly on the available phase space. For a given initial condition we follow the dynamical trajectory. Since the Lyapunov exponent is positive, numerical accuracy is lost after $9-30$ collisions depending on the value of $R$. For $R$ very close to $1 / \sqrt{2}$ times up to even 100 collisions can be reached. (We refer to [14] for an extensive discussion on numerical issues and on error estimates for billiards.) The viscosity is determined from a linear least square fit over the last 5-10 collision times. Since the fit turns out to be essentially perfect, we expect to be in the asymptotic regime already. This could be checked only by following the trajectories over even longer times which is not feasible with accuracy. In Fig. 6 we show the viscosities for the parameters given in Sect. 3. Note the different scales.

The shear viscosity increases with $R$, the denser the fluid the stronger the internal friction, and diverges as $(1 / \sqrt{2}-R)^{-3 / 2}$ for $R \rightarrow 1 / \sqrt{2}$, as can be seen from the insert in Fig. 6a. We understand this behavior by arguing as follows. Let $\delta=1 / \sqrt{2}-R$ and let us divide $[0, t]$ into small intervals of length $t_{0}$ with $t_{0}$ of the order of one collision time. Then 

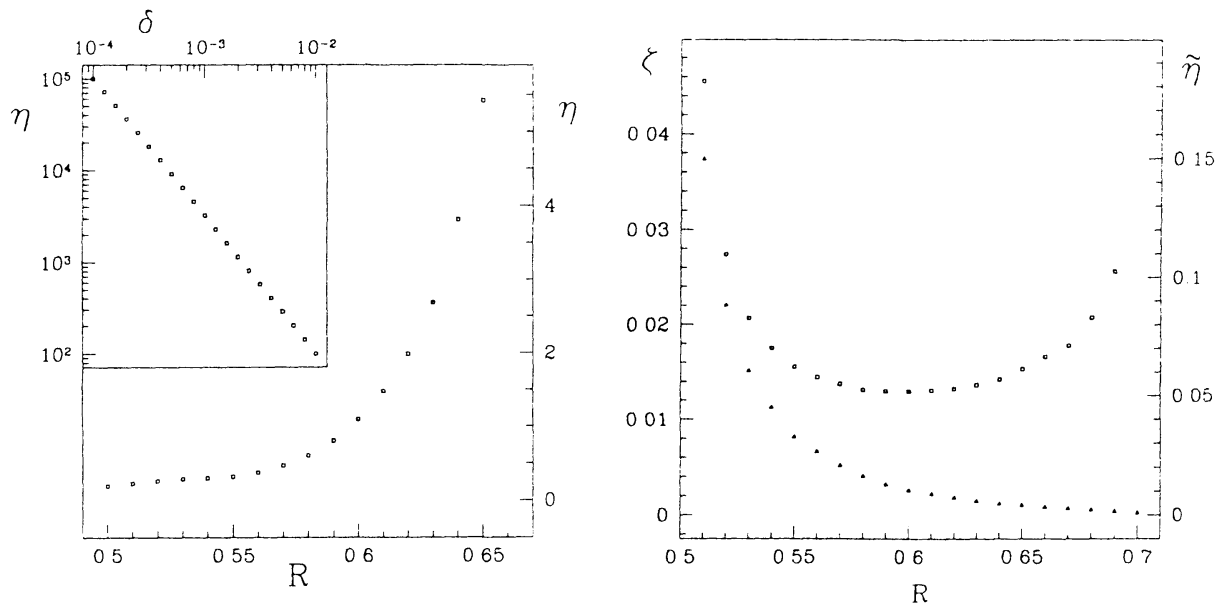

Fig. 6a,b. (a) Shear viscosity $\eta(\square)$ aganst hard disk diameter $R$. The insert is a logarithmic plot at $R=(1 / \sqrt{2})-\delta$. (b) Bulk viscosity $\zeta(\square)$ and viscosity $\tilde{\eta}(\triangle)$ against hard disk radius $R$.

$$
\begin{aligned}
\frac{1}{t}\left\langle\tau_{3}([0, t])^{2}\right\rangle & =\frac{1}{t} \sum_{i=0}^{t / t_{0}} \sum_{j=0}^{t / t_{0}}\left\langle\tau_{3}\left(\left[i t_{0},(i+1) t_{0}\right]\right) \tau_{3}\left(\left[j t_{0},(j+1) t_{0}\right]\right)\right\rangle \\
& \cong \frac{1}{t_{0}} \sum_{j}\left\langle\tau_{3}\left(\left[0, t_{0}\right]\right) \tau_{3}\left(\left[j t_{0},(j+1) t_{0}\right]\right)\right\rangle
\end{aligned}
$$

We assume that

$$
\left\langle\tau_{3}\left(\left[0, t_{0}\right]\right) \tau_{3}\left(\left[j t_{0},(j+1) t_{0}\right]\right)\right\rangle \cong\left\langle\tau_{3}\left(\left[0, t_{0}\right]\right)^{2}\right\rangle \exp \left[-|j| \lambda t_{0}\right] .
$$

Here $\lambda t_{0}$ is the Lyapunov exponent measured in number of collisions. Now $t_{0} \simeq \delta$, $\left\langle\tau_{3}\left(\left[0, t_{0}\right]\right)^{2}\right\rangle \simeq 1$ independent of $\delta$, and $\lambda t_{0} \simeq \sqrt{\delta}[2]$. This yields the scaling exponent $3 / 2$. Presumably, the bulk viscosity diverges also as $R \rightarrow 1 / \sqrt{2}$. However for $R$ close to $1 / \sqrt{2}$, the quantity $\left\langle\tau_{1}([0, t])^{2}\right\rangle$ starts to oscillate and $\zeta$ can no longer be determined meaningfully. A similar problem arises at $R=0.5$.

It is tempting to compare the $N=2$ results with those for larger systems $[1,13]$. To do so we first have to write the viscosity in dimensionless form. Let us return to (2.8) in case of $N$ hard (hyper-) spheres with mass $m$ and diameter $a$ on a $d$-torus of linear dimension $l$, volume $V=l^{d}$. Their kinetic energy, $E$, is identified with the temperature by $E=N \frac{d}{2} k_{B} T$. The physical viscosity tensor, $\mathscr{Z}$, is then

$$
\mathscr{V}_{\alpha \beta, \gamma \delta}=V^{-1} \frac{d N}{4 E} D_{\alpha \beta, \gamma \delta},
$$

compare with (2.13). As usual we define the reduced density by

$$
\rho^{*}=\rho / \rho_{c p}=N \sqrt{3 / 2 d}(a / l)^{d},
$$

where $\rho_{c p}$ is the density of close packing. Clearly, $\mathscr{L}$ depends nontrivially only on $\rho^{*}$ and $N$. By scaling space, time, and mass we obtain 


$$
\begin{aligned}
\mathscr{L}_{\alpha \beta, \gamma \delta}(m, E, V, A ; N)= & a^{-(d-1)} \sqrt{\frac{m E}{N}}\left(\frac{d}{2}\right) \\
& \times\left[\rho^{*(d-1) / d} \mathscr{V}_{\alpha \beta, \gamma \delta}\left(1, N, \sqrt{3 / 2 d} N, \rho^{* 1 / d} ; N\right)\right] .
\end{aligned}
$$

By symmetry, the term in the square bracket depends only on the dimensionless viscosities $\eta^{*}, \zeta^{*}, \tilde{\eta}^{*}$, which are functions of the reduced density $\rho^{*}$ and the number $N$ of particles. The dimensionless viscosities should have a limit as $N \rightarrow \infty$, provided $d \geq 3$, and $\eta^{*}=\tilde{\eta}^{*}$ in the limit, as explained before. For $d=2$ one expects an anomalous volume dependence and $\mathscr{C}$, as defined, should diverge for (very) large $N$.
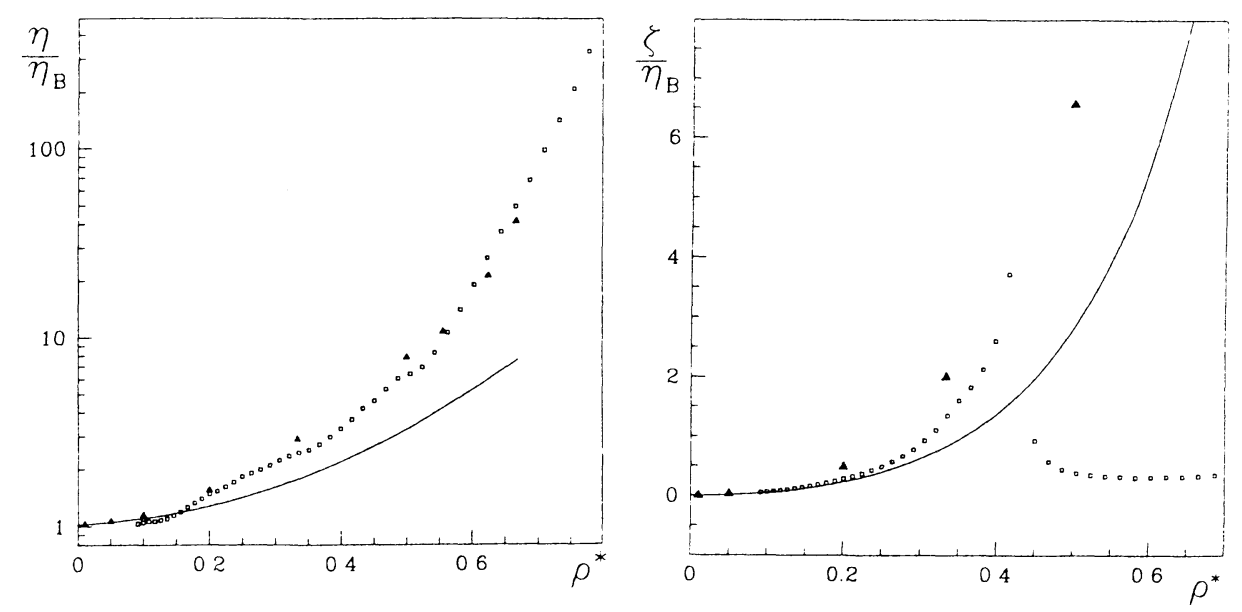

Fig. 7a,b. (a) Shear viscosity $\eta(\square)$ and (b) bulk viscosity $\zeta(\square)$ against reduced density $\rho^{*}$. - is the Enskog approximation and $\triangle$ are the data from Ref.1. The value $\zeta=1.2 \pm 0.3$ at $\rho^{*}=0.433(R=0.5)$ has been omitted. The vertical scale is in units of the $d=2$ Boltzmann shear viscosity $\eta_{B}$.

In Fig. 7 we compare our data $(N=2, d=2)$ with molecular dynamics for $d=3$, $N=108,500,4000[1,13]$. (No numerical results for $d=2$ seem to be available.) We also plot the predictions of the $d=2$ Enskog theory. It is based on the assumption that the stress correlation is an exact exponential, which can be determined then from its value and its derivative at $t=0$. It is convenient to plot $\eta, \zeta$ in units of the $d=2$ Boltzmann shear viscosity $\eta_{B}=(2 \sqrt{\pi} a)^{-1} \sqrt{k_{B} T m}=(2 \sqrt{\pi} a)^{-1} \sqrt{m E / N}$. A comparison with (7.5) shows that $\eta / \eta_{B}$ and $\zeta / \eta_{B}$ are dimensionless and depend only on $\rho^{*}$ and $N$. We emphasize that Fig. 7 contains no fit parameters.

There is one sublety. Theorem 1 covers only reduced densities $0.433 \leq \rho^{*} \leq$ 0.866 . For the shear viscosity the $45^{\circ}$ channels open up at $\rho^{*}=0.217$. Thus for the bulk viscosity $\zeta^{*}$ with $\rho^{*} \leq 0.433$ and the shear viscosity $\eta^{*}$ with $\rho^{*} \leq 0.217$ we expect for the variance an intermediate regime linear in $t$ which eventually crosses over to a $t \log t$ behaviour. The length of this linear regime should increase with $\rho^{*}$. Because of the restricted time span available the logarithmic correction cannot be seen numerically. Note that the Lyapunov exponent is decreasing with $\rho^{*}$, which even further restricts the available time span. E.g. at $\rho^{*}=0.108(R=0.25)$ we can follow the trajectories only up to 9 collisions. The fraction of trajectories with 0 collision is then 0.018 whereas the fraction with 0,1 collisions is 0.026 . At $\rho^{*}=0.351$ 
( $R=0.45$ ) we can follow the trajectories already up to 16 collisions. The fraction with 0 collisions is then $2.8 \cdot 10^{-3}$ and with 0,1 collisions $4.1 \cdot 10^{-3}$. On this basis we hope that the numerical viscosities remain meaningful even for $\rho^{*} \leq 0.433$.

Acknowledgement. We are grateful to N. I. Chernov for very helpful discussions and suggestions. H. S. thanks H. van Beijeren for providing us with tables on the Enskog viscosity. L. B. acknowledges the support of the National Science Foundation, Grant DMS-9303769.

\section{References}

1. Alder, B. J., Gass, D. M., Wainwright, T. E.: Studies in molecular dynamics. VIII. The transport coefficients for a hard-sphere fluid. J. Chem. Phys. 53, 3813-3826 (1970)

2. Benettin, G.: Power law behaviour of Lyapunov exponents in some conservative dynamical systems. Physica D13, 211-213 (1984)

3. Bleher, P. M.: Statistical properties of two-dimensional periodic Lorentz gas with infinite horizon. J. Stat. Phys. 66, 315-373 (1992)

4. Bunimovich, L. A.: Decay of correlations in dynamical systems with chaotic behavior. J. Exp. Theor. Phys. 89, 1452-1471 (1985)

5. Bunimovich, L. A.: Dynamical systems of hyperbolic type with singularities. In: Sinai, Ya. G. (ed.) Encycl. Math. Sci. Dynamical Systems. Vol. 2, Berlin, Heidelberg: Springer-Verlag, 1989, pp. 151-179

6. Bunimovich, L. A., Sinai, Ya. G.: The fundamental theorem in the theory of dispersed billiards. Math. USSR-Sb. 19, 407-423 (1973)

7. Bunimovich, L. A., Sinai, Ya. G.: Markov partition of dispersed billiards. Commun. Math. Phys. 78, 247-280 (1980); Erratum 107, 357-358 (1986)

8. Bunimovich, L. A., Sinai, Ya. G.: Statistical properties of the Lorentz gas with periodic configuration of scatterers. Commun. Math. Phys. 78, 479-497 (1981)

9. Bunımovich, L. A., Sinai, Ya. G., Chernov, N. I.: Markov partitions of two-dimensional billiards. Russ. Math. Surv. 45, 105-152 (1990)

10. Bunimovich, L. A., Sinai, Ya. G., Chernov, N. I.: Statistical propertıes of two-dimensional hyperbolic billiards. Russ. Math. Surv. 46, 47-106 (1991)

11. Chernov, N. I.: Statistical properties of the periodic Lorentz gas. Multidimensional case. J. Stat. Phys. 74, 11-53 (1994)

12. Chernov, N. I.: Limit theorems and Markov approximations for chaotic dynamical systems. Probability Theory and Related Fields (to be published)

13. Erpenbeck, J. J., Wood, W. W.: Molecular dynamics techniques for hard core systems. In: Berne, B. J. (ed.) Statistical Mechanics. Modern Theoretical Chemistry. Vol. 6, New York: Plenum Press, 1977 , pp. $1-40$

14. Garrido, P., Gallavotti, G.: Billiard correlation functions. J. Stat. Phys. 76, 549-585 (1994)

15. Ibragimov, I. A., Linnik, Yu. V.: Independent and stationary sequences of random variables. Groningen: Wolters, Noordhoff, 1971

16. Knauf, A.: Ergodic and topological properties of coulombic periodic potentials. Commun. Math. Phys. 109, 1-24 (1987)

17. McLennan, J. A.: Introduction to nonequilibrium statistical mechanics. Englewood Cliffs: Prentice Hall, 1989

18. Presutti, E.: A mathematical definition of the thermodynamic pressure. J. Stat. Phys. 13, 301-314 (1975)

19. Sinai, Ya. G.: Dynamical systems with elastic reflections. Ergodic properties of dispersing billiards. Russ. Math. Surv. 25, 137-189 (1970)

20. Sinai, Ya. G.: Ergodic properties of the Lorentz gas. Funct. Anal. Appl. 13, 46-59 (1979)

21. Spohn, H.: Large scale dynamics of interacting particles. Berlin, Heidelberg, New York: Springer, 1991

\section{Communicated by Ya. G. Sinai}

This article was processed by the author using the Springer-Verlag TEX CoMaPhy macro package 1991. 